\title{
The Occurrence of Dementia in Alzheimer disease and Vascular Dementia in the Republic of Belarus
}

\author{
T. Dakukina, T. Golubeva, T. Korotkevich, A. Startsev, E. Sheremet \\ Republican scientific-practical center of mental health, Department of mental and \\ behavioral disorders- section science, Minsk, Belarushis
}

Objectives:

The purpose of the study was to analyze the rates of dementias, including those of dementia in Alzheimer disease and vascular dementia, in the Republic of Belarus.

Background:

The percentage of older people in Belarus in increasing, resulting in growing number of reported cases of mental diseases, in particular with dementias.

Materials and Methods:

National statistical data for 2017 were used to obtain absolute numbers of cases and to calculate the occurrence rates (per 100,000 people).
Results and Conclusions:

By the end of 2017, 10,142 people in Belarus were registered as having dementia and requiring regular supervision by the doctor, including 1,609 people (15.9\%) with dementia in Alzheimer disease and 4,612 people (45.5\%) with vascular dementia. The total occurrence of dementia was 202.5 per 100,000 people, including 28.3 per 100,000 people for dementia in Alzheimer disease and 98.5 per 100,000 people for vascular dementia. The rate of hospitalization with dementias was 4.6 per 10 thousand population, including 0.6 per 10 thousand for dementia in Alzheimer disease and $\mathbf{2 . 2}$ per 10 thousand for vascular dementia.

The above results demonstrate the prevalence of vascular dementia over the dementia in Alzheimer disease in the overall pattern of dementias in population of Belarus.The obtained data show the urgent need for improvement of diagnostic methods to differentiate between the dementia types and prevent the cerebrovascular diseases. 